\title{
Systematic Study of \\ Spectrometer-Induced Azimuthal Asymmetries for SpinQuest
}

FERMILAB-SLIDES-20-103-E

Forhad Hossain

with

Stephen Pate, Vassili Papavassiliou, and Abinash Pun

On behalf of the SpinQuest Collaboration

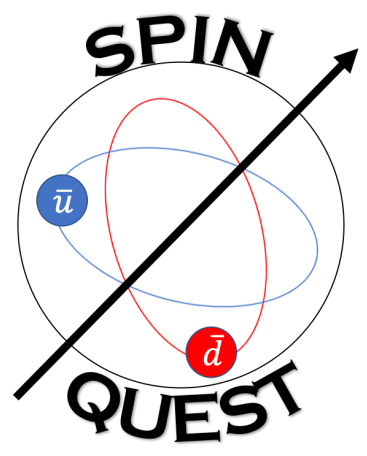




\section{Outline}

1. Transverse Single-Spin Asymmetry.

2. Sivers Effect in the Nucleon.

3. Sea-quark Sivers Asymmetry from Polarized Drell-Yan.

4. Extracting the Spectrometer-Induced Azimuthal Asymmetry.

5. Summary and Conclusions. 


\section{Transverse Single-Spin Asymmetry}

W.H. Dragoset et al., PRL36, 929 (1976)

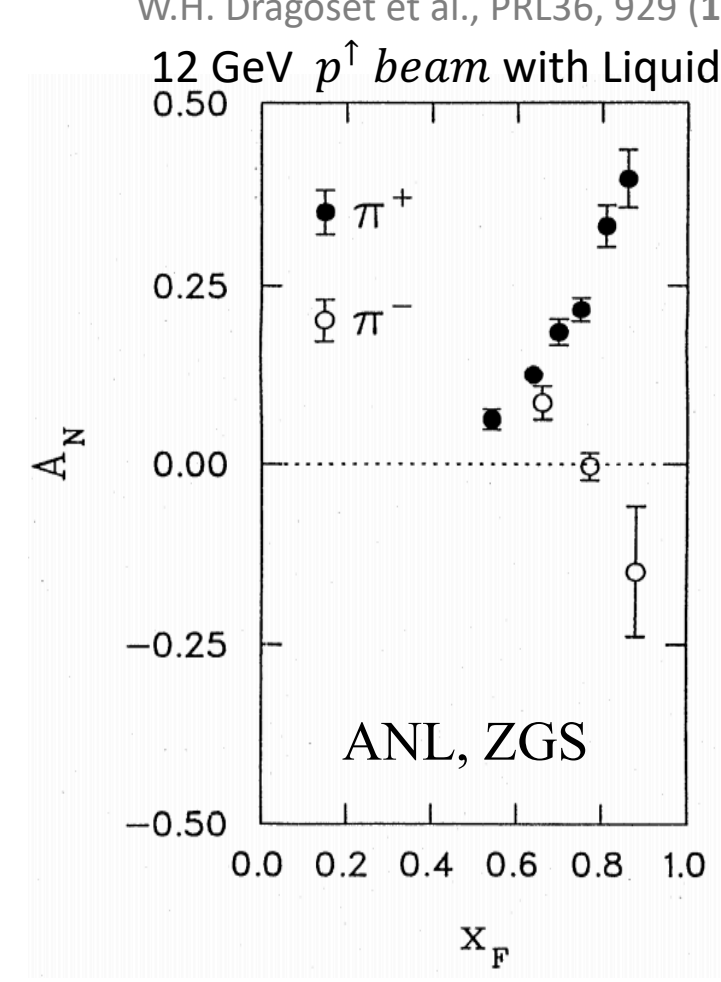

\section{How it all started?}
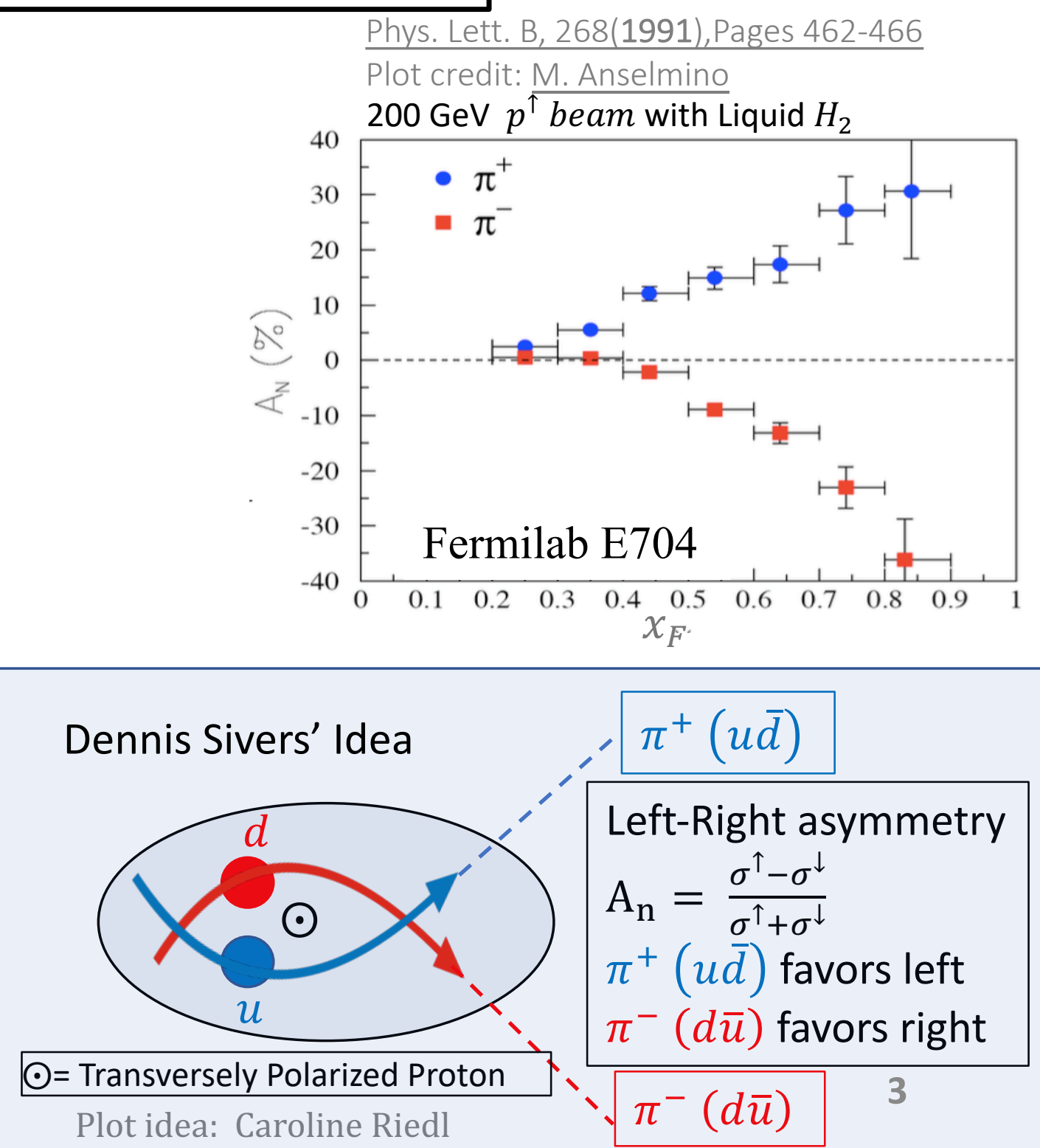


\section{Sivers Effect in the Nucleon}

\section{Reasons for the Asymmetry}

The number density of unpolarized quarks in a transversely polarized proton:

Phys. Rev. D 70, 117504 (2004) Phys. Rev. D 67, 074010 (2003)

$$
f_{q / p^{\uparrow}}\left(x, \vec{k}_{T}\right)=f_{1}^{q}\left(x, k_{T}^{2}\right)-f_{1 T}^{\perp q}\left(x, k_{T}^{2} \frac{\left(\vec{k}_{T} \times \vec{S}\right) \cdot \vec{P}}{m_{p}}\right.
$$

Dennis Sivers, Phys. Rev. D 41, 83 (1990)

The $\vec{k}_{T}$ distribution of quarks in a transversely polarized Gives correlation between $\vec{k}_{T}$ and $\vec{S}$ proton can be asymmetric and known as Sivers effect.

$\mathrm{f}_{1}^{\mathrm{q}}=$ Unpolarized quark density.

$f_{1 T}^{\perp q}\left(x, k_{T}^{2}\right)=$ Sivers function.

$x=$ Bjorken variable.

$\vec{S}=$ Spin polarization vector.

$\vec{P}=$ Three momentum of the proton.

$\vec{k}_{T}=$ Intrinsic transverse momentum of unpolarized quarks.
Sivers Effect: Intrinsic $k_{T}$ imbalance leads to the asymmetry
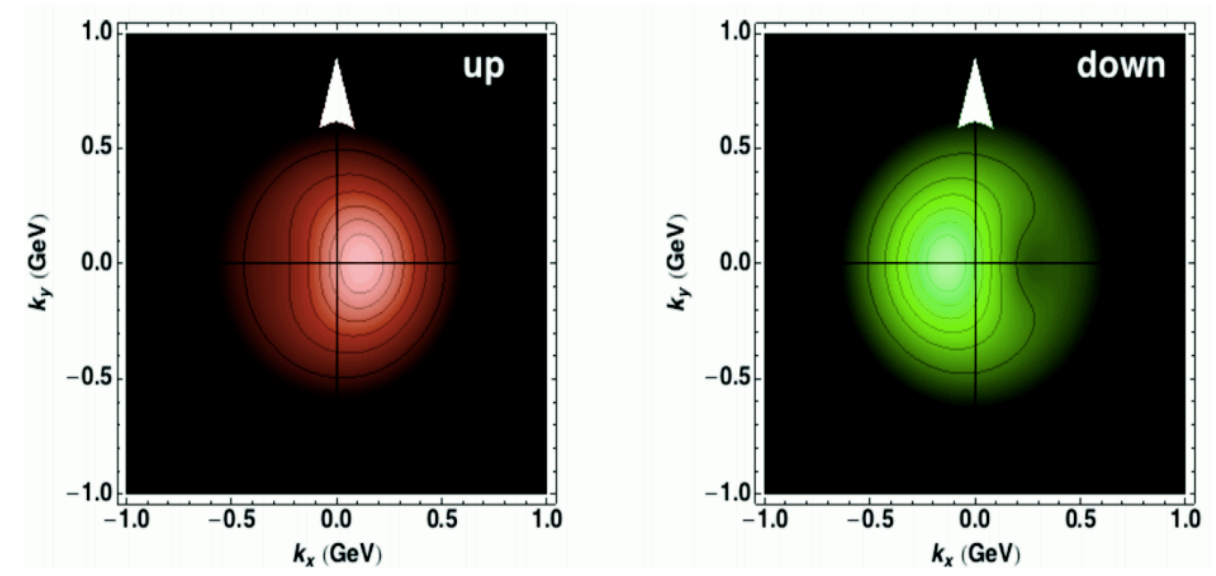

Bacchetta \& Contalbrigo, The proton in 3D II Nuovo Saggiatore 28 (12) n.1,2 


\section{Sea-quark Sivers Asymmetry from Polarized Drell-Yan}

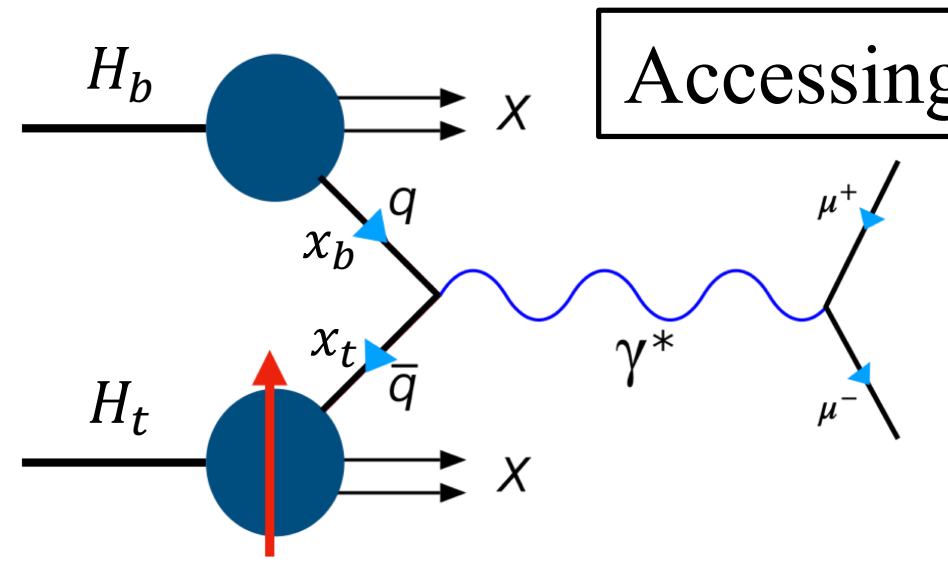

1. Bjorken variable $x$ is the fractional longitudinal momentum carried by the scattered parton.

2. The sea quarks and gluons PDF dominate at low $x$.

3. Valence quarks dominate at high $x$.

4. large- $x$ sea quarks and low- $x$ valence quarks are suppressed. Hence most interactions come from high $x$ valence and low $x$ sea quarks.

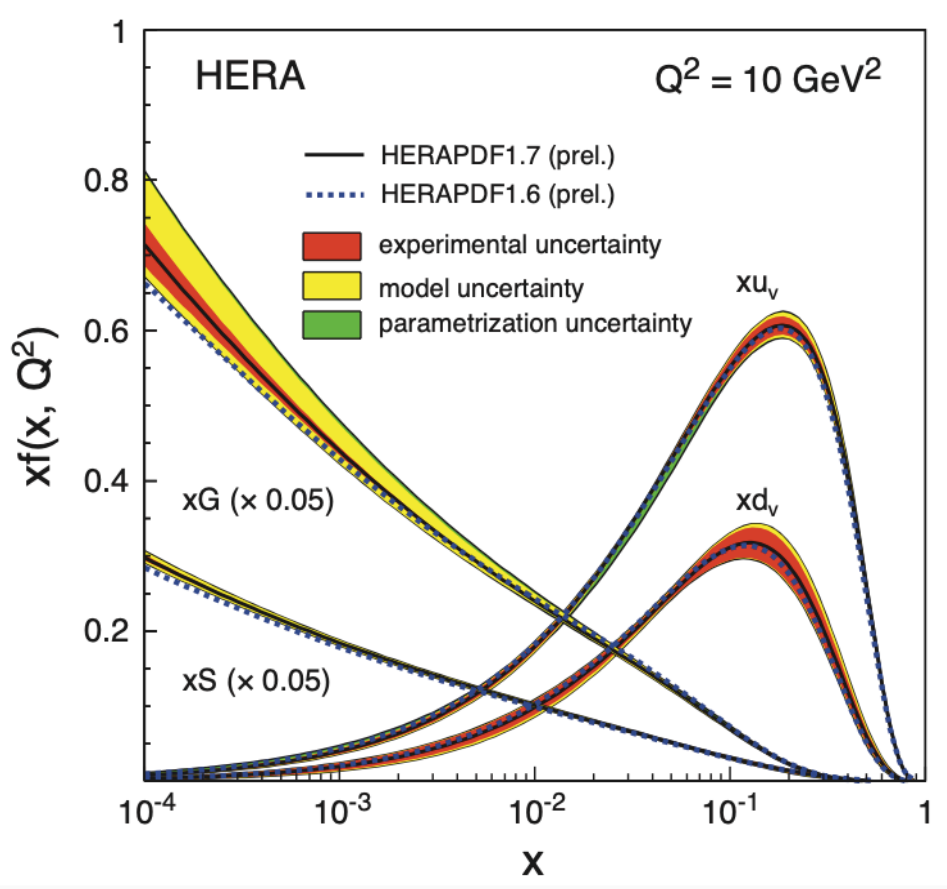

Fig: Proton parton distribution functions plotted as functions of Bjorken $\mathrm{x}$

$$
\frac{d^{2} \sigma}{d x_{b} d x_{t}}=\frac{4 \pi \alpha^{2}}{9 x_{b} x_{t}} \frac{1}{s} \sum_{q} e_{q}^{2}\left(q_{b}\left(x_{b}\right) \bar{q}_{t}\left(x_{t}\right)+q_{t}\left(x_{t}\right) \bar{q}_{b}\left(x_{b}\right)\right)
$$

1. For SpinQuest $x_{b}>x_{t}$

2. Kinematic acceptance plot in next slide 5 


\section{Sea-quark Sivers Asymmetry from Polarized Drell-Yan}

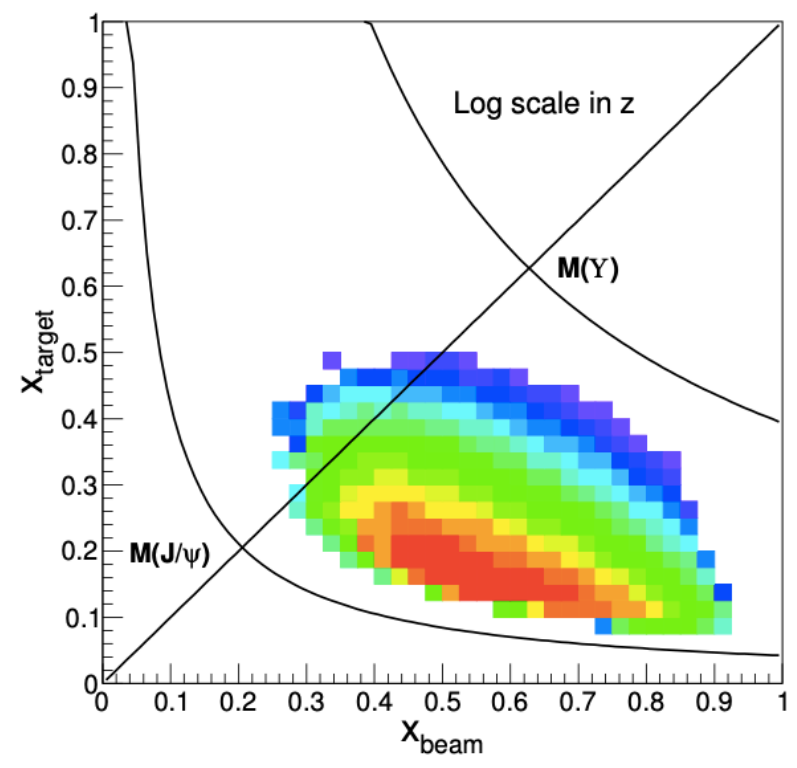

\section{SpinQuest Apparatus}

The kinematic acceptance

of the SpinQuest experiment. $\left(x_{b}>x_{t}\right)$

Transversely Polarized $\mathrm{NH}_{3}$ or $\mathrm{ND}_{3}$.

$\frac{\text { Main Injector Beam }}{120 \mathrm{GeV} \text { Proton }}$

$300 \mathrm{~cm}$ Upstream Face

$(x, y, z)=(0,0,0)$

seaQuest Dimuon spectrometer

1. Tracking : Abinash Pun

2. FPGA Trigger: MinJung Kim

3. Polarization: Zulkaida Akbar

4. Dilution Factor: Anchit Arora 


\section{Sea-quark Sivers Asymmetry from Polarized Drell-Yan}

The Drell-Yan Cross Section in Terms of Sivers Asymmetry:

$$
\begin{aligned}
& \sigma_{D Y}^{\uparrow \downarrow}=\frac{d \sigma^{L O}}{d^{4} q d \phi_{S}} \propto 1 \pm\left|\mathrm{S}_{\mathrm{T}}\right| \sin \phi_{S} A_{T}^{\sin \phi_{S}} \\
& A\left(\phi_{S}\right)=\frac{1}{\left|S_{T}\right|} \frac{\sigma_{D Y}^{\uparrow}-\sigma_{D Y}^{\perp}}{\sigma_{D Y}^{\uparrow}+\sigma_{D Y}^{\perp}}=\sin \phi_{S} A_{T}^{\sin \phi_{S}}
\end{aligned}
$$

\begin{tabular}{|l|}
$\vec{S}_{T}=$ Target spin vector \\
$\widehat{x}, \widehat{y}, \widehat{z}$, is target rest frame $=\mathrm{TF} ; \hat{x}=\hat{q}_{T}, \hat{y}=\hat{z} \times \hat{q}_{T}$ \\
$\hat{x}^{\prime}, \hat{y}^{\prime}, \hat{z}^{\prime}$ is detector rest frame $=\mathrm{DF}$ \\
$\vec{q}_{T}=$ Dimuon's transverse momentum \\
$\vec{k}_{T}=$ Quark's transverse momentum \\
\hline
\end{tabular}

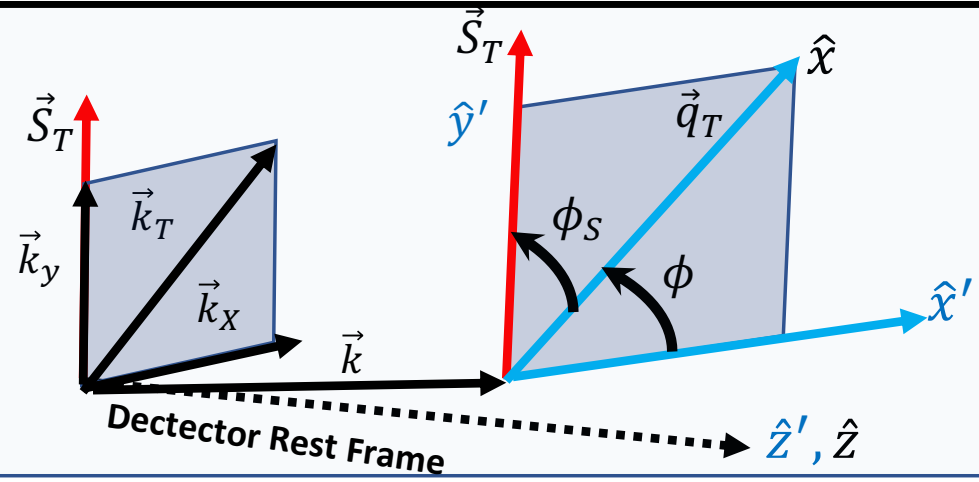

Phys. Rev. D 79, 034005 (2009),

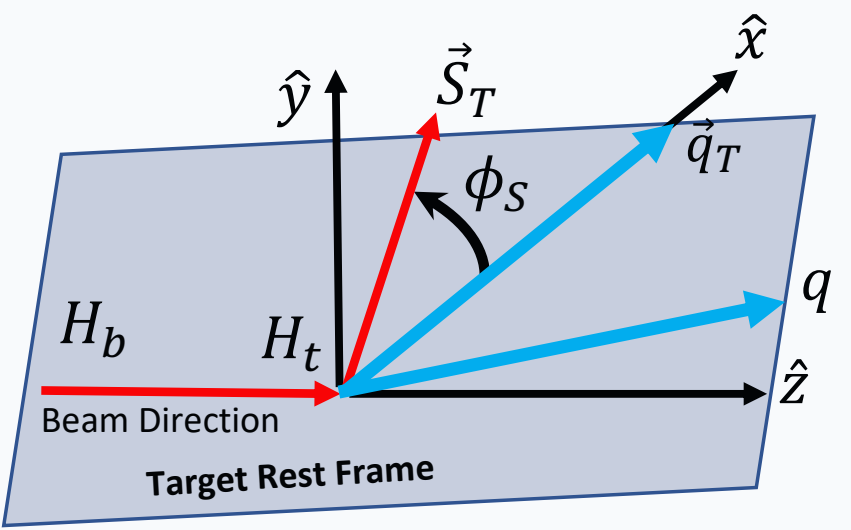

1. $\sigma_{D Y}^{\uparrow \downarrow}$ is the Drell-Yan cross section and $A_{T}^{\sin \phi_{S}}$ is the Sivers asymmetry .

2. Azimuthal angle $\phi_{S}$ in TF and $\phi$ in DF can be written as $\phi_{S}^{\uparrow \downarrow}=\left( \pm \frac{\pi}{2}-\phi\right)$. 


\section{Extracting the Spectrometer-Induced Azimuthal Asymmetry}

Motivation for the Spectrometer-Induced Asymmetry

Systematic Check

1. The efficiency of the detectors could be changing over time (e.g. the phototubes might suffer a decrease in gain).

2. The numbers of protons hitting the polarized target will not be the same in each spill, and the detector performance could change due to luminosity fluctuations.

$\rightarrow$ In either case, it is possible for one target spin state to collect a higher yield than the other, producing a false asymmetry. We will check the size of such effects, by using the SeaQuest experiment dataset. 


\section{Extracting the Spectrometer-Induced Azimuthal Asymmetry}

Dimuon Yield $\left(N_{\uparrow \downarrow}\right)$ and Fake Spin Assignment in SeaQuest Data

$A_{\mathrm{n}}=\frac{\frac{\mathrm{N}_{\uparrow}}{\mathrm{L}_{\uparrow}^{\text {int }}}-\frac{N_{\downarrow}}{\mathrm{L}_{\downarrow}^{\text {int }}}}{\frac{\mathrm{N}_{\uparrow}}{\mathrm{L}_{\uparrow}^{\text {int }}}+\frac{N_{\downarrow}}{\mathrm{L}_{\downarrow} \text { int }}}=S_{T} A(\phi)$

$$
N_{\uparrow \downarrow}(\phi)=L_{\uparrow \downarrow}^{i n t} \Omega \epsilon_{\mathrm{T}} \sigma\left[1 \pm S_{T} A(\phi)\right]
$$
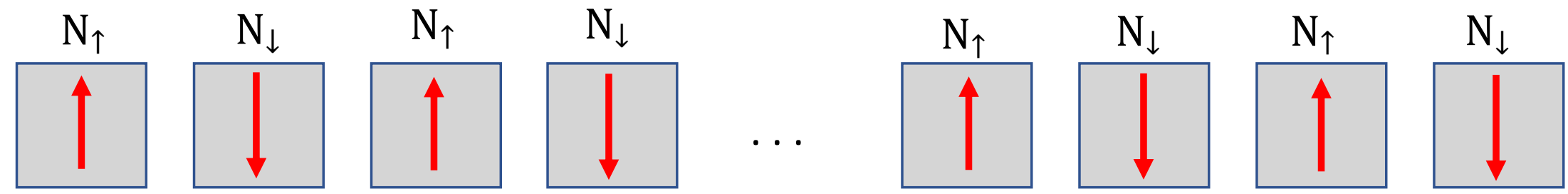

Beginning of the Spin flipping

1. $\sigma$ is the unpolarized interaction cross section.

2. $\Omega$ is the overall acceptance of the spectrometer.

3. $L_{\uparrow \downarrow}^{i n t}$ is the integrated number of protons hitting the target $L H_{2}$ when DAQ is live.

4. $\mathrm{S}_{\mathrm{T}}$ is the transverse polarization. We have assumed 1 for the (fake) spin flipping case.

5. $\epsilon_{\mathrm{T}}$ overall efficiency of the measurement.

6. $A(\phi)$ can have the form of the detector false asymmetry or physics asymmetry Sivers. 


\section{Extracting the Spectrometer-Induced Azimuthal Asymmetry \\ Flipping Spin Every Hour}

1. Standard SeaQuest selections was used where the dimuon mass was $4.2 \mathbf{G e V}$ or more.

2. Total protons on $\mathrm{LH}_{2}$ target is $\mathbf{1 . 5} \times \mathbf{1 0}^{\mathbf{1 7}}$ and total DAQ runs are analyzed is about $\sim \mathbf{3 0 0 0}$.

3. Each run is about one hour and only $\mathrm{LH}_{2}$ target was used from the runs.

4. The fitting function $\operatorname{par}[\mathbf{0}]+\operatorname{par}[\mathbf{1}] * \cos \boldsymbol{\phi}$ is used with least-squares method.

5. The asymmetry $\operatorname{Par}[1]=-0.0002 \pm 0.0090$

6. From the results we do not see any false spectrometer generated asymmetry produced.

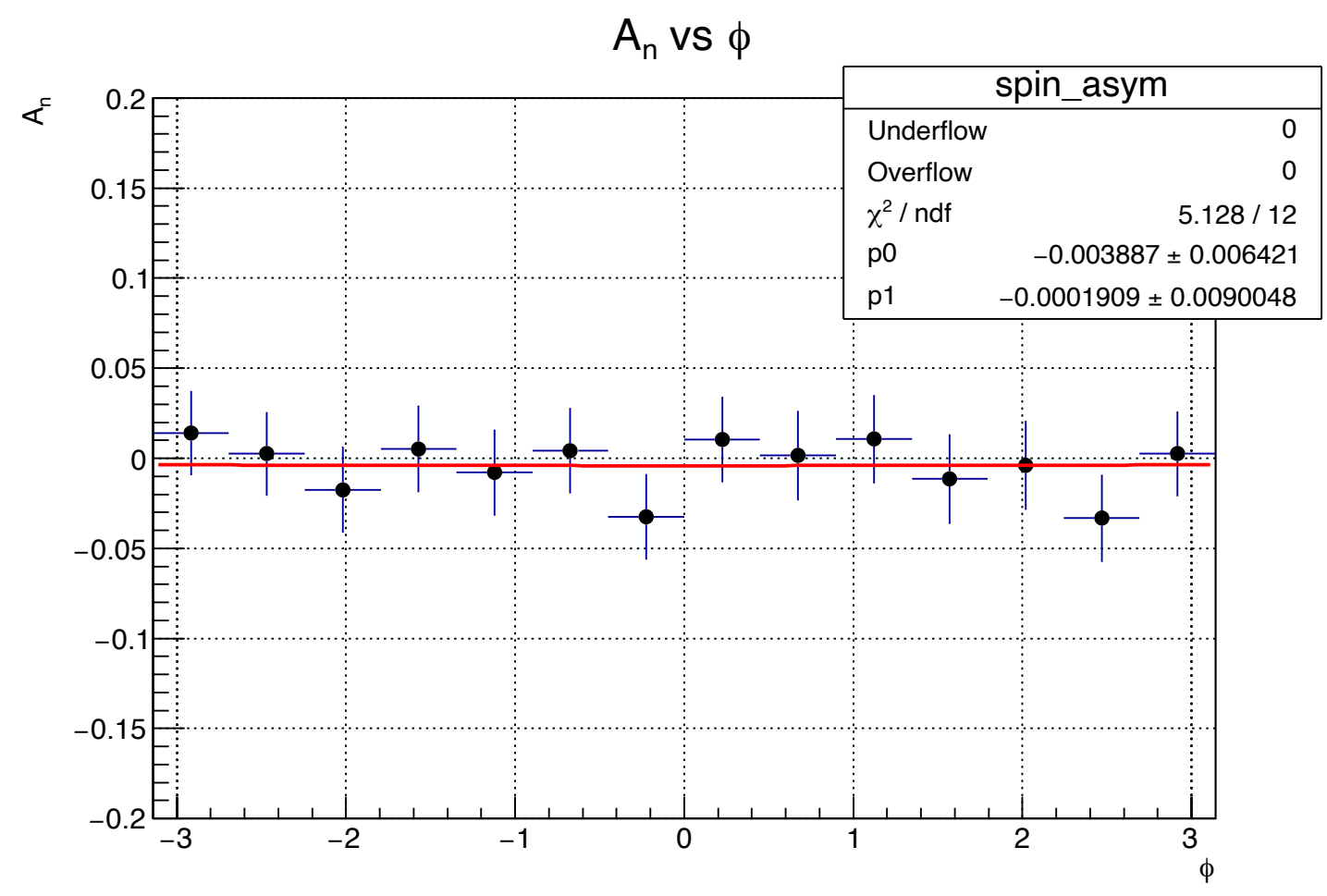




\section{Extracting the Spectrometer-Induced Azimuthal Asymmetry}

\section{Flipping Spin Every 12 Hours (Day/Night, a Worst-Case Scenario)}

1. Standard SeaQuest selections was used where the dimuon mass was $4.2 \mathrm{GeV}$ or more.

2. Total protons on $\mathrm{LH}_{2}$ target is $1.5 \times 10^{17}$ and total DAQ runs are analyzed is about $\sim 3000$.

3. Each run is about one hour and only $\mathrm{LH}_{2}$ target was used from the runs.

4. The fitting function $\operatorname{par}[0]+\operatorname{par}[1] * \cos \phi$ is used with least-squares method.

5. The asymmetry $\operatorname{Par}[1]=-0.007 \pm 0.009$

6. From the results we do not see any false spectrometer generated asymmetry produced.
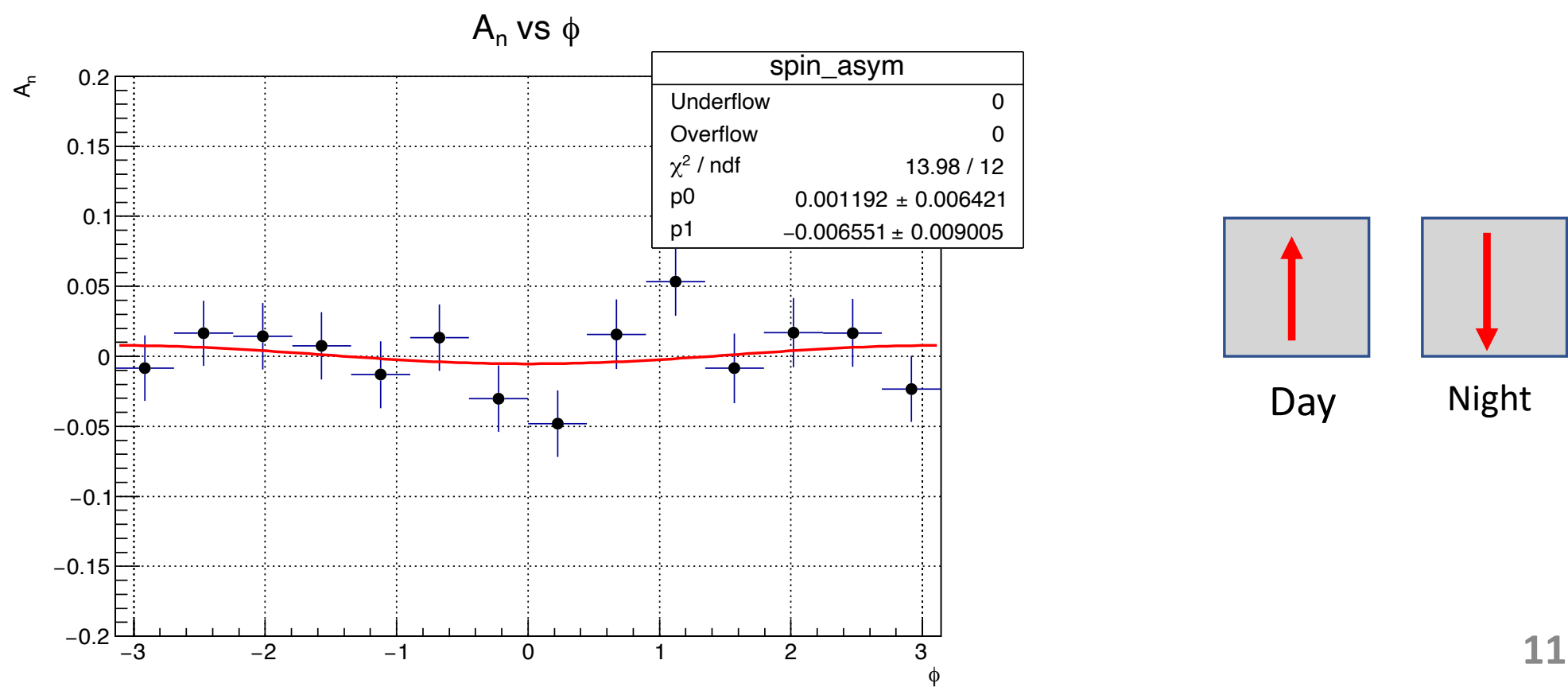


\section{Extracting the Spectrometer-Induced Azimuthal Asymmetry}

\section{Flipping Spin Every 50 Hour (a Reasonable Scenario)}

1. Standard SeaQuest selections was used where the dimuon mass was $4.2 \mathrm{GeV}$ or more.

2. Total protons on $\mathrm{LH}_{2}$ target is $1.5 \times 10^{17}$ and total DAQ runs are analyzed is about $\sim 3000$.

3. Each run is about one hour and only $\mathrm{LH}_{2}$ target was used from the runs.

4. The fitting function $\operatorname{par}[0]+\operatorname{par}[1] * \cos \phi$ is used with least-squares method.

5. The asymmetry $\operatorname{Par}[1]=-0.001 \pm 0.009$

6. From the results we do not see any false spectrometer generated asymmetry produced.

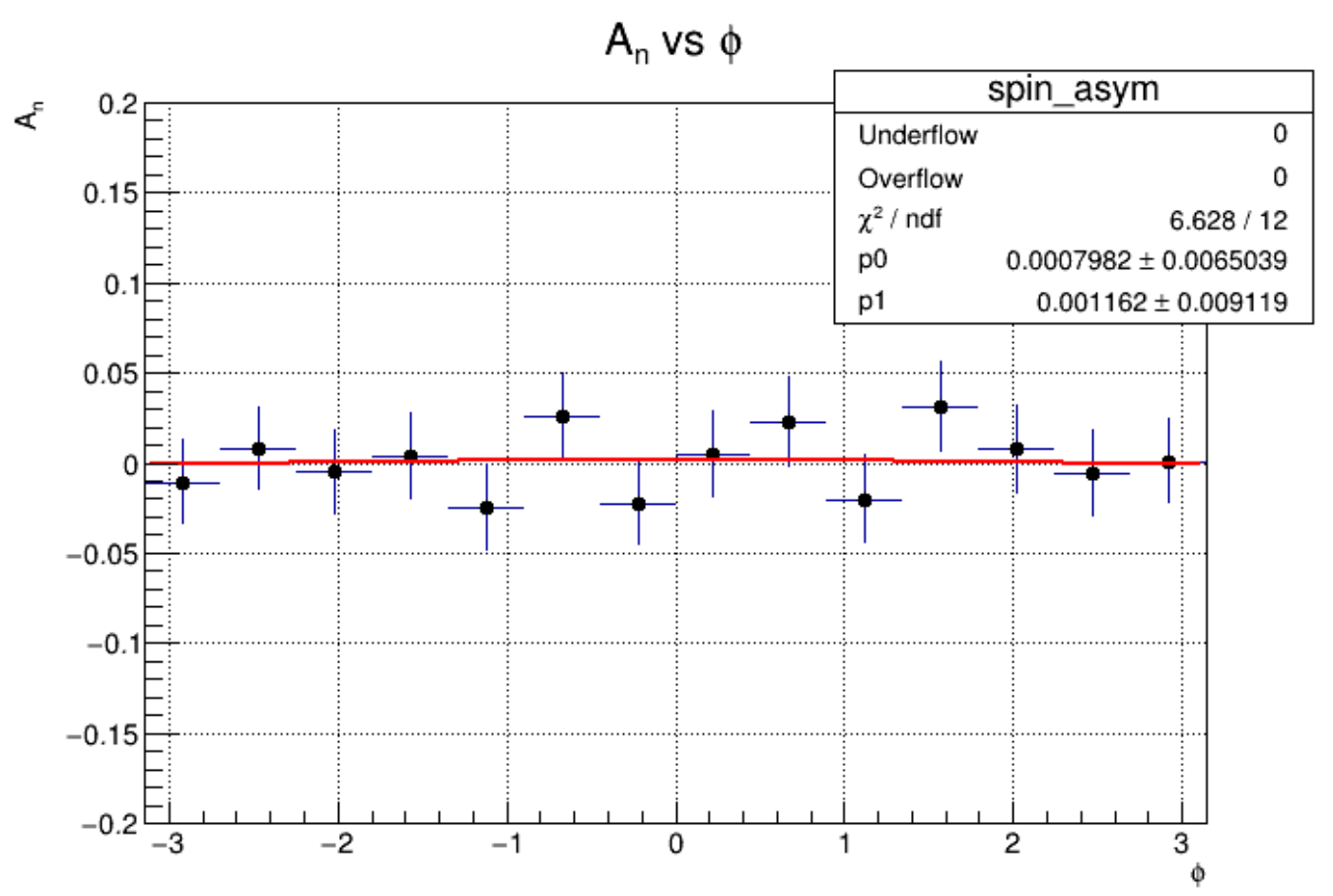




\section{Summary and Conclusions}

- We have done a systematic study of measuring the fake azimuthal asymmetries that could be introduced due to the spectrometer.

- We have found that the detector did not generate a false azimuthal asymmetry that could imitate the physics Sivers asymmetry.

Thanks to all SeaQuest collaborators, especially Kenichi Nakano and Andrew Chen for all the suggestions and comments in the study. 


\section{Back Up Slides}




\section{Sivers Effect in the Nucleon}

\section{Quarks Distributions in Impact Parameter Space}

A transversely polarized proton deformation is described by the gradient of the Fourier transform of $E_{q}\left(x, \xi=0,-\Delta_{\perp}^{2}\right)$.

M. Burkardt, Few-Body Syst 52, 265-270 (2012) $q\left(x, \mathbf{b}_{\perp}\right)=\int \frac{d^{2} \Delta_{\perp}}{(2 \pi)^{2}} H^{q}\left(x, 0,-\Delta_{\perp}^{2}\right) e^{-i \mathbf{b}_{\perp} \cdot \Delta_{\perp}}-\frac{1}{2 M} \frac{\partial}{\partial b_{y}} \int \frac{d^{2} \Delta_{\perp}}{(2 \pi)^{2}} E^{q}\left(x, 0,-\Delta_{\perp}^{2}\right) e^{-i \mathbf{b}_{\perp} \cdot \mathbf{\Delta}_{\perp}}$ $d_{y}^{q} \equiv \int d^{2} b_{\perp} q\left(x, b_{\perp}\right) b_{y}=\frac{\boldsymbol{k}_{q}}{2 M} \quad ;$ Positive (negative) drift for the up (down) quark

\section{Burkardt, Int.J.Mod.Phys.A 18 (2003) 173-208}

Anomalous magnetic moments of the quarks [considering the $u$ and d quarks only]

- $\kappa_{p}=\frac{2}{3} \kappa_{u}-\frac{1}{3} \kappa_{d}=1.79$

- $\kappa_{n}=\frac{2}{3} \kappa_{d}-\frac{1}{3} \kappa_{u}=-1.91$

- $\kappa_{u}=1.673 ; \quad \kappa_{d}=-2.033$

IMF Frame

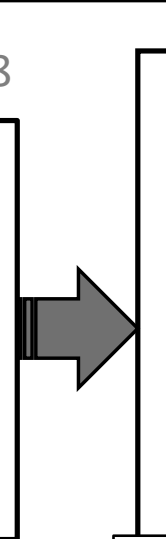

$u_{X}\left(\mathrm{x}, \mathrm{b}_{+}\right)$

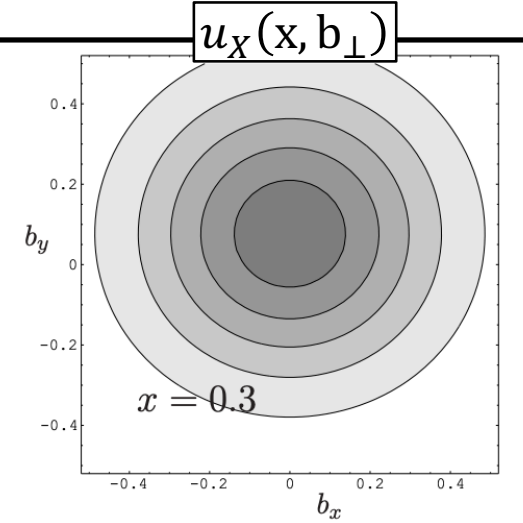

Quarks distributions in impact parameter space, where the proton is transversely polarized in $\mathrm{x}$ direction.

The reason $\mathrm{u}$ and $\mathrm{d}$ quarks are drifting in opposite directions is due to the anomalous magnetic moment of the quarks to the proton. 


\section{Time Dependent Spectrometer-Induced Asymmetry}

1. Each segment consists of range of 100 DAQ runs. If any runs are missing in any segment, we will have less than 100 runs in that case.

2. Polynomial fit pol0 to the $F=\frac{\mathrm{N}_{\uparrow}}{\mathrm{N}_{\downarrow}} \times \frac{\mathrm{L}_{\downarrow}}{\mathrm{L}_{\uparrow}}$ is consistent with 1 , and we also have 0 asymmetry with time.
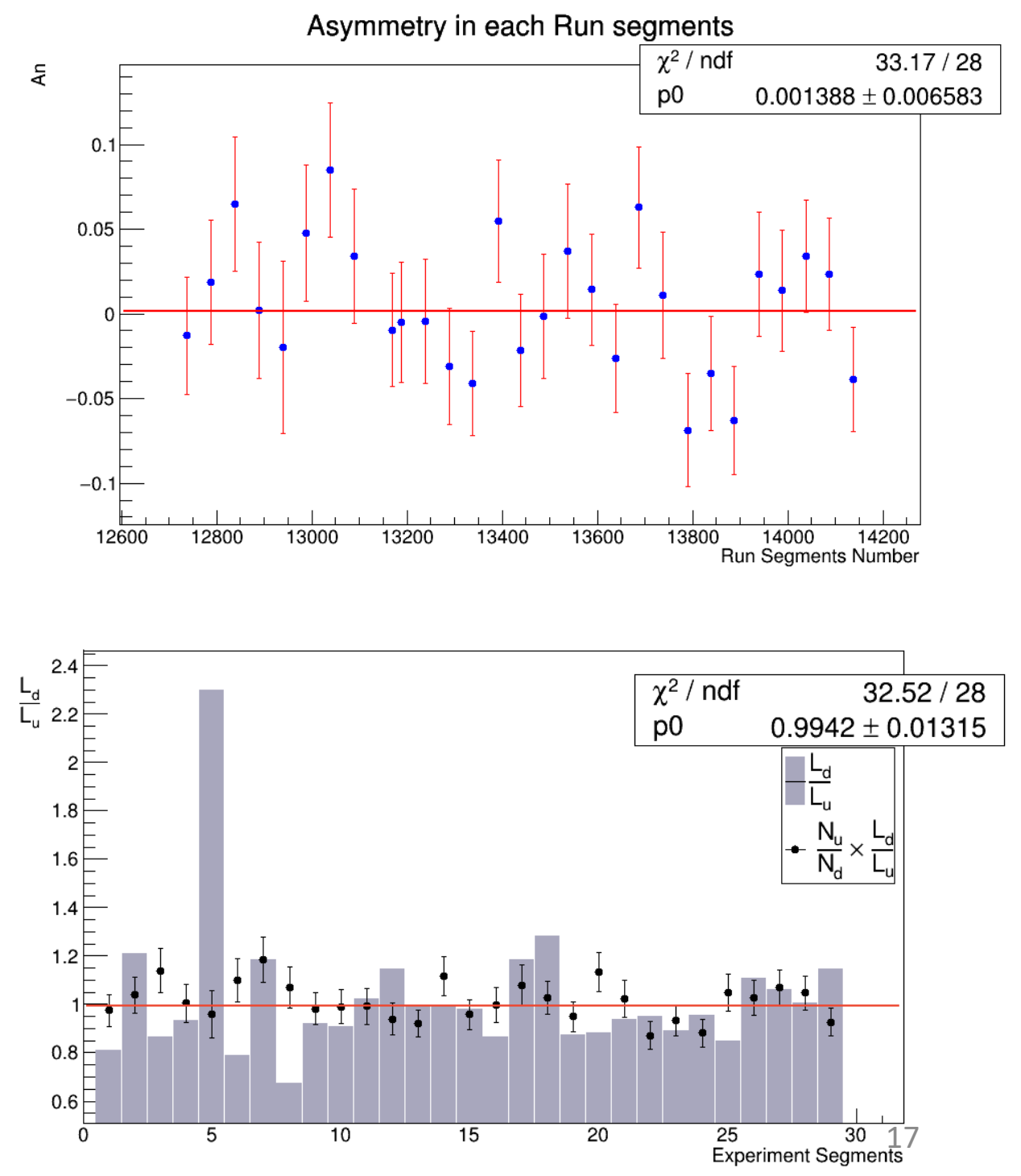


\section{Sea-quark Sivers asymmetry from polarized Drell-Yan}

\section{What is the Drell-Yan Process?}

VOLUMe 25, Number 21

PHYSICAL REVIEW LETTERS

23 NOVEMBER 1970

Observation of Massive Muon Pairs in Hadron Collisions*

J. H. Christenson, G. S. Hicks, L. M. Lederman, P. J. Limon, and B. G. Pope

Columbia University, New York, New York 10027, and Brookhaven National Laboratory, Upton, New York 11973

and

E. Zavattini

CERN Laboratory, Geneva, Switzerland

(Received 8 September 1970)

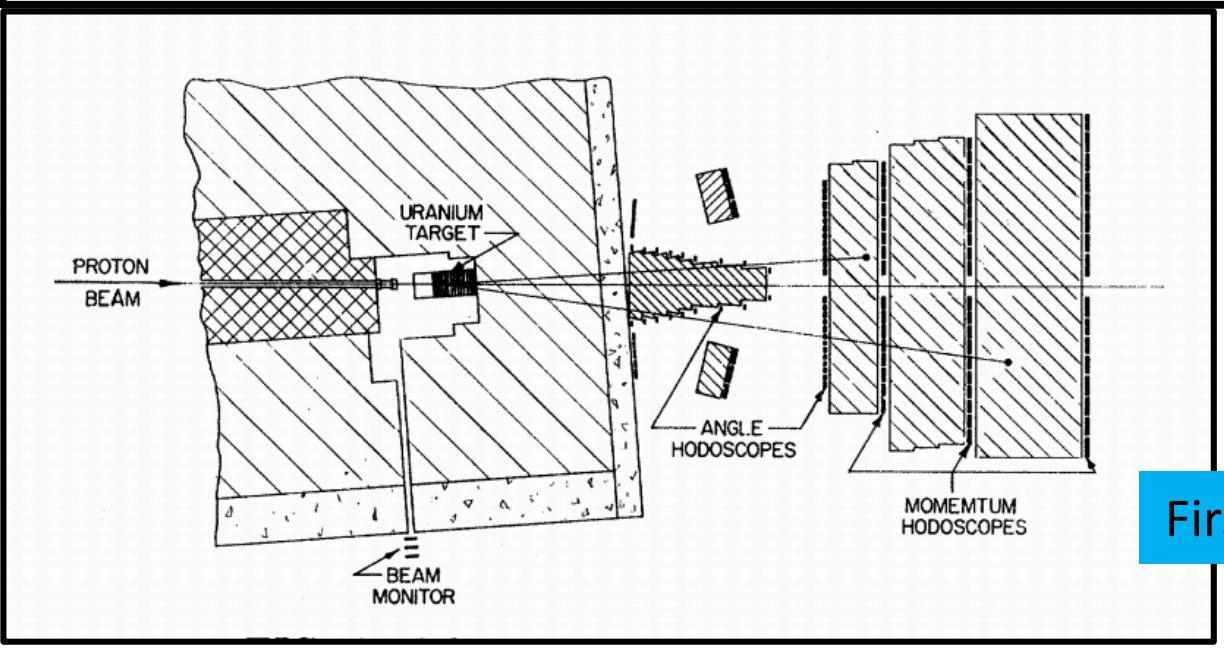

\section{First Explained}

Sidney D. Drell and Tung-Mow Yan

Phys. Rev. Lett. 25, 902 (1970)

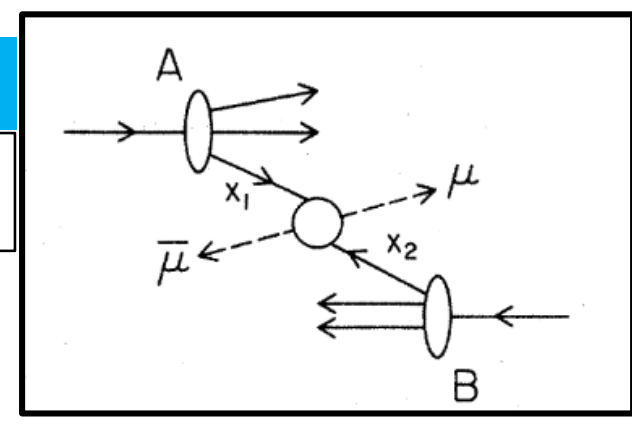

First Ovserved

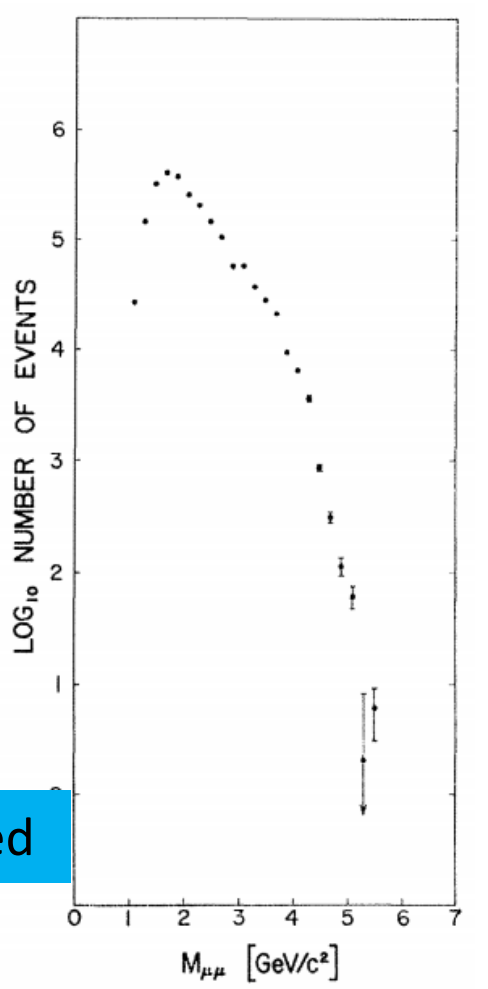




\section{Drell Yan Cross Section Formula \&}

\section{Azimuthal Asymmetry}

The leading order transversely single Drell-

Yan cross section in QCD parton model:
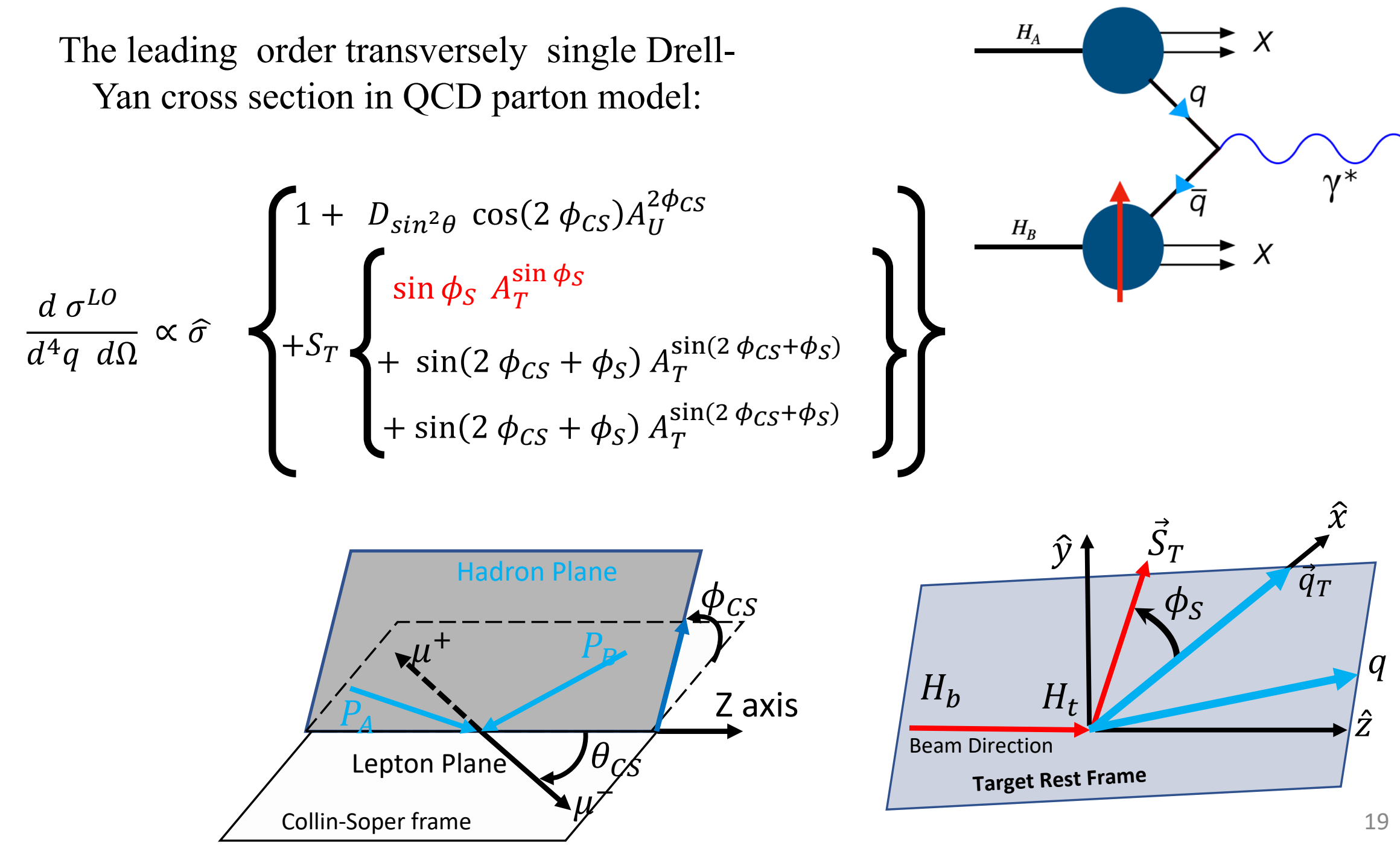


\section{Sea-quark Sivers Asymmetry from Polarized Drell-Yan}

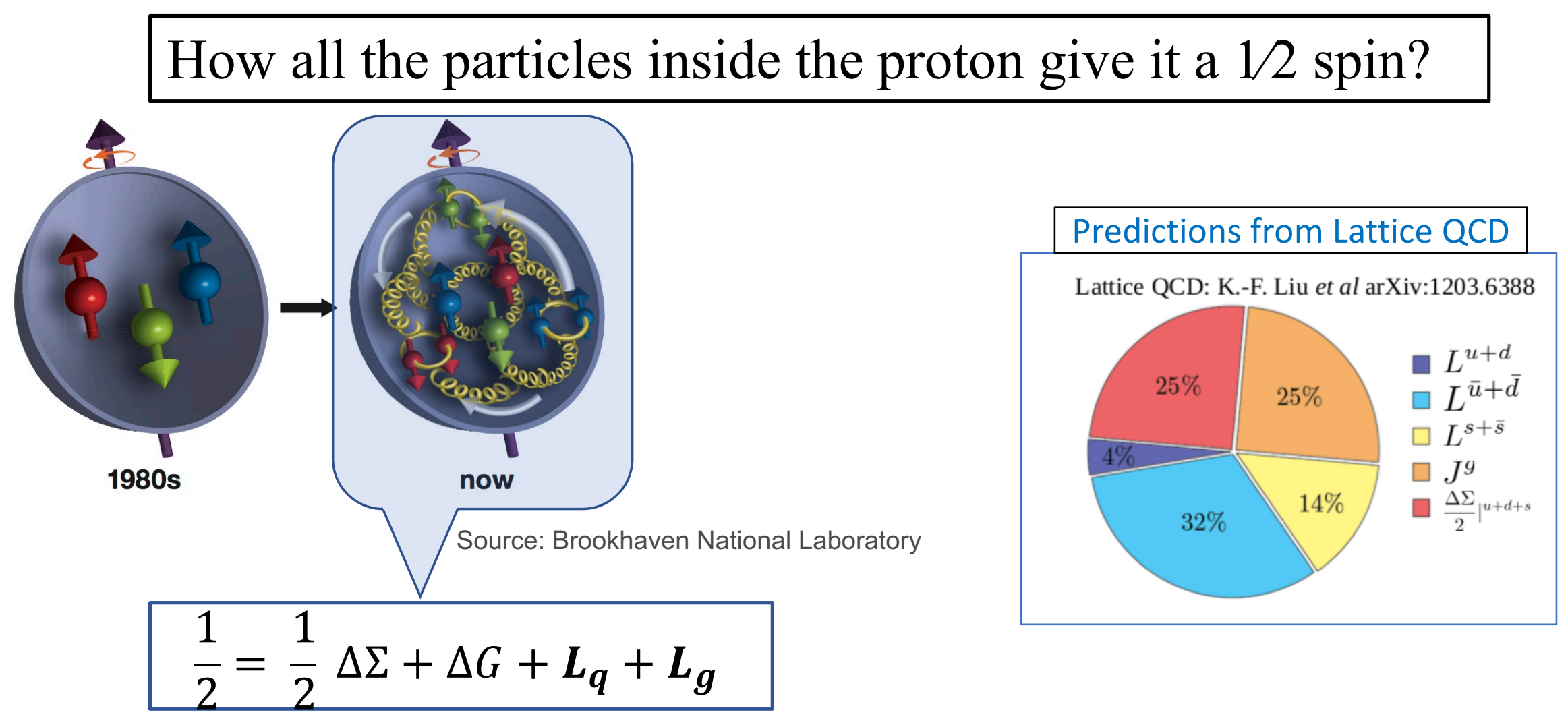

1. Quarks spin accounts for about $25 \%$ of the proton's spin.

2. From recent study of RHIC, it is still disputed that the sum of both quark and gluon spin contributions make up the total proton spin.

3. Lattice QCD indicates that $\sim \mathbf{5 0} \%$ comes from the quark's orbital angular momentum.

4. Hence, orbital angular momentum of sea quarks could play major role in proton ${ }_{2}$ pin. Hints of sea quark O.A.M. 


\section{Sea-quark Sivers Asymmetry from Polarized Drell-Yan}

Anticipated Results from SpinQuest

$$
\text { If } A_{\text {Siv }} \neq 0
$$

1. If the Sivers asymmetry $A_{S I V} \neq 0$, we will find a strong evidence that orbital momentum of sea quarks are not zero.

2. We will determine the Sivers function as well.

$$
\text { If } A_{\text {Siv }}=0
$$

Flavor asymmetry of sea quarks would be more difficult to understand.

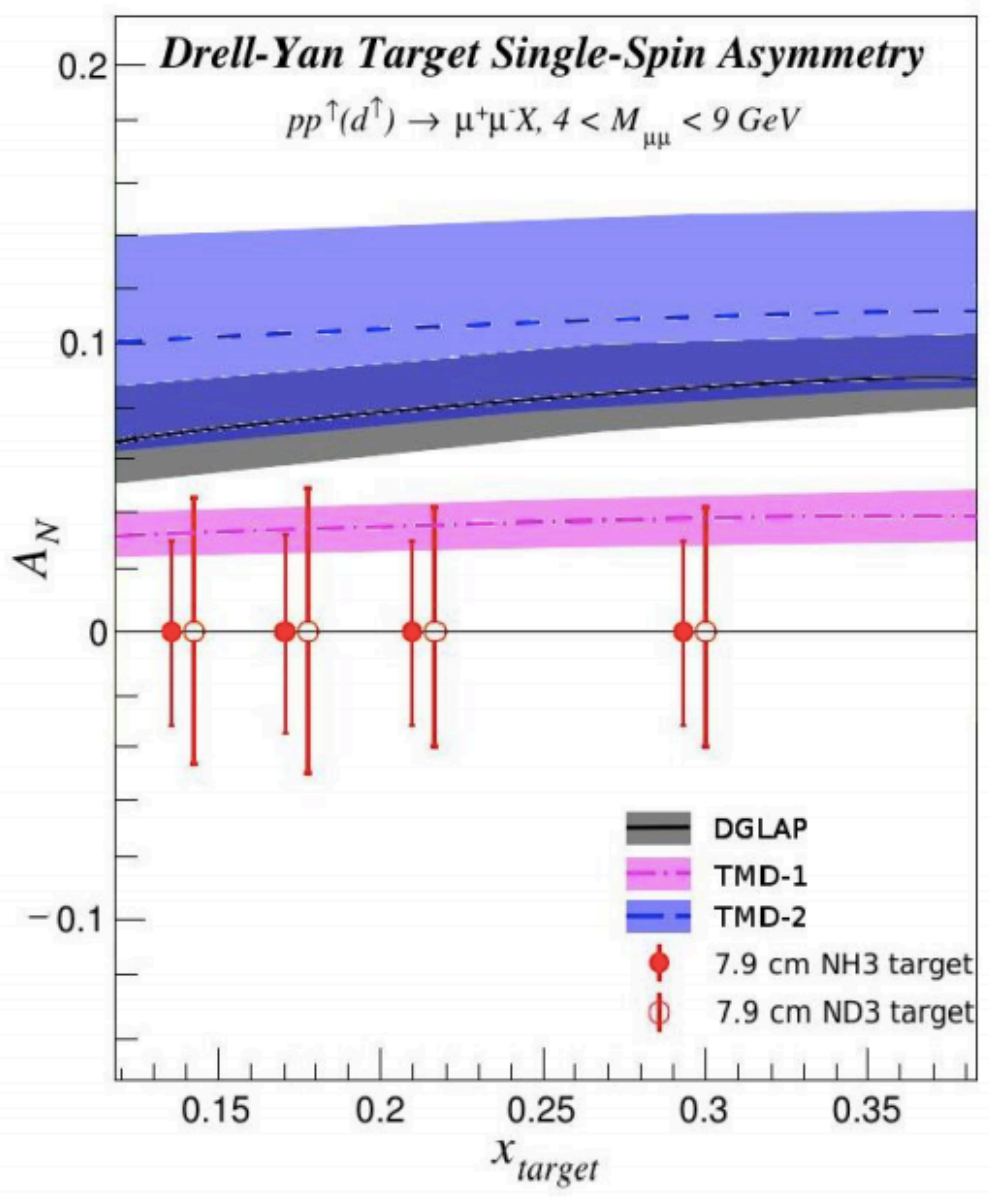

DGLAP: M. Anselmino et al arXiv:1612.06413 TMD-1: M. G. Echevarria et al arXiv:1401.5078 TMD-2: P. Sun and F. Yuan arXiv:1308.5003

The anticipated uncertainties in the SpinQuest experiment are based on combined running on $\mathrm{NH}_{3}$ and $\mathrm{ND}_{3}$ targets. 